\title{
Considerations for Effective Low Power Wireless Sensor Systems in Industrial Environments
}

\author{
Almholt, Thomas \\ Grazier, Mark \\ Texas Instruments \\ Dallas, Texas USA
}

\begin{abstract}
This paper addresses key considerations for enabling robust low power wireless sensor links for use in large scale industrial settings. Applications include large, multi-story buildings that have complicated layouts and use building materials such as metal, reinforced concrete, and corrugated metal plates which are not friendly to RF communication systems. We will review wireless communication theory and discuss how to enable robust and reliable communication in these types of environments. We will review a generic RF communication channel and then offer techniques to limit or eliminate typical interferers to radio systems. We begin by the reviewing most frequently observed impairments in a radio channel. Next we will present techniques to combat each of these impairments.

We then proceed to the typical system level techniques to improve radio performance like CSMA (listen before talk), automatic acknowledgments, receive signal strength indicators, antenna diversity and forward error correction. We will conclude with a discussion of how advanced transceiver functionality and fast RF settling times enable ultra low power wireless sensors to be powered by energy harvesters for solar cells and piezo-electrics devices.
\end{abstract}

\section{Frequency selectivity in static environment:}

A wireless channel is a space and frequency dependant, time variant system. This means that the instantaneous performance of the communication channel will change with frequency, time and physical changes. To obtain a reliable wireless communication link, the wireless system should incorporate techniques that convert these challenges into benefits. For instance, changing the frequency of operation is called "frequency diversity," changing placement in position becomes "spatial diversity," and performing a retry becomes "time diversity."

Frequency selectivity happens when an RF signal passes thru an environment with multiple possible paths from transmitter to receiver a so-called "multipath channel." Multipath channels are found in almost every environment except deep space - in fact, on the surface of the earth there will almost always be two or more ways an RF signals can travel from start to finish. In outdoor urban environments a signal will typically find one or more building walls to reflect off, thereby creating a multipath channel. In indoor environments walls, floors and ceilings provide excellent reflectors. At the receiver antenna a linear combination of all signals occurs, creating constructive or destructive interference. The level of constructive and destructive interference is dependant on the quality of the reflectors - that is the "quality" walls, ceilings and floors. This is a function of surface permittivity, conductivity and smoothness. Typical urban buildings in the US are constructed of a wood frame, plaster board and a brick finish. Even these non-conducting surfaces create substantial multipath reflections at $2.4 \mathrm{GHz}$ causing deep fades as illustrated below. It is found that variations in signal strength of more than $30 \mathrm{~dB}$ occurs across a frequency range of 2400 to $2475 \mathrm{MHz}$, which was repeatable at least 5 times over a 12 second interval. Furthermore, it is found that the two receivers did not see the same RF channel. In this particular 
case RSSI1 and RSSI2 seem to be somewhat correlated, but offset in frequency by roughly $10 \mathrm{MHz}$ and the deepest fades is less than $4 \mathrm{MHz}$ wide.

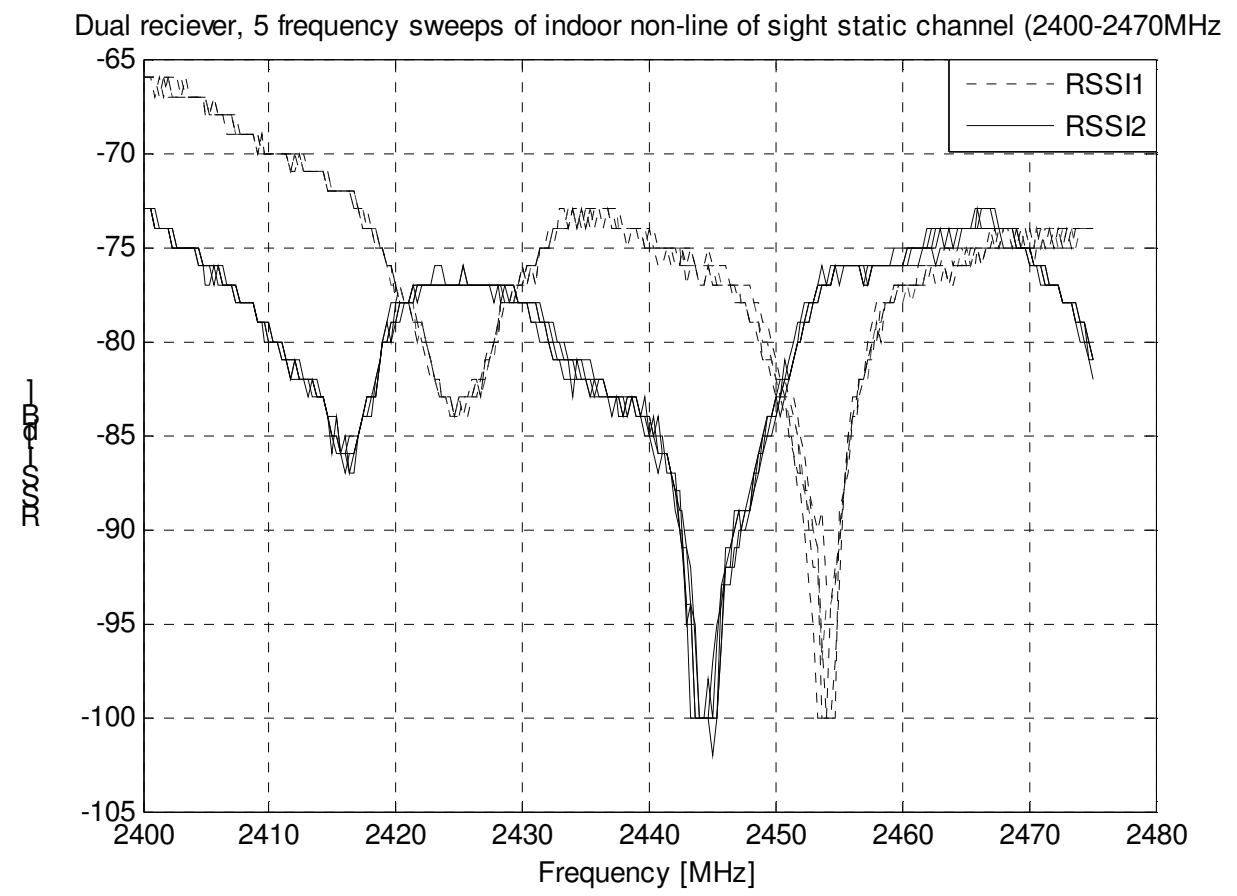

Figure 1 Indoor non-line of sight in a typical urban house using one transmitter and two receivers. The two receivers are spaced $15 \mathrm{~cm}$ apart and connected to the same microcontroller. The output frequency is swept from 2400 to $2475 \mathrm{MHz}$ in 225 steps. The channel is swept five times where each sweep takes 2.25 seconds.

Therefore we can conclude that it is very important for static environments to include some kind of mechanism to avoid these deep fades in the frequency domain. The two best approaches become frequency hopping spread spectrum or antenna diversity based on spatial diversity as illustrated in Figure 1.

- Frequency hopping requires no additional hardware, but it does add more complexity in the MAC layer in the form of a complicated scheduler which is needed to keep all the nodes aligned. Bluetooth ${ }^{\mathrm{TM}}$ is based on this type of diversity with its 1600 hops per second across 79 channels.

- Antenna diversity does require more hardware but in many cases can be very cost effective, if factors like lifetime power consumption is considered. Many DECT cordless telephone implements some type of antenna diversity.

Between the two options there is not a clear winner, designers must consider the overall system and make a choice on a case by case basis.

\section{Multipath fading in dynamic environments}

Figure 2 shows how multipath fading effects a single channel while moving slowly around inside the channel. This is represented using a simple measurement of Received Signal Strength (RSSI) for two receivers spaced approximately $15 \mathrm{~cm}$ apart receiving the same exact burst of data from a single transmitter. Each burst of data lasts approximately $2 \mathrm{~ms}$ and a new burst of data is transmitted every $10 \mathrm{~ms}$, the location of the transmitter is changed at a pace of $0.5 \mathrm{~m} / \mathrm{s}$ equivalent to a slow walk. 


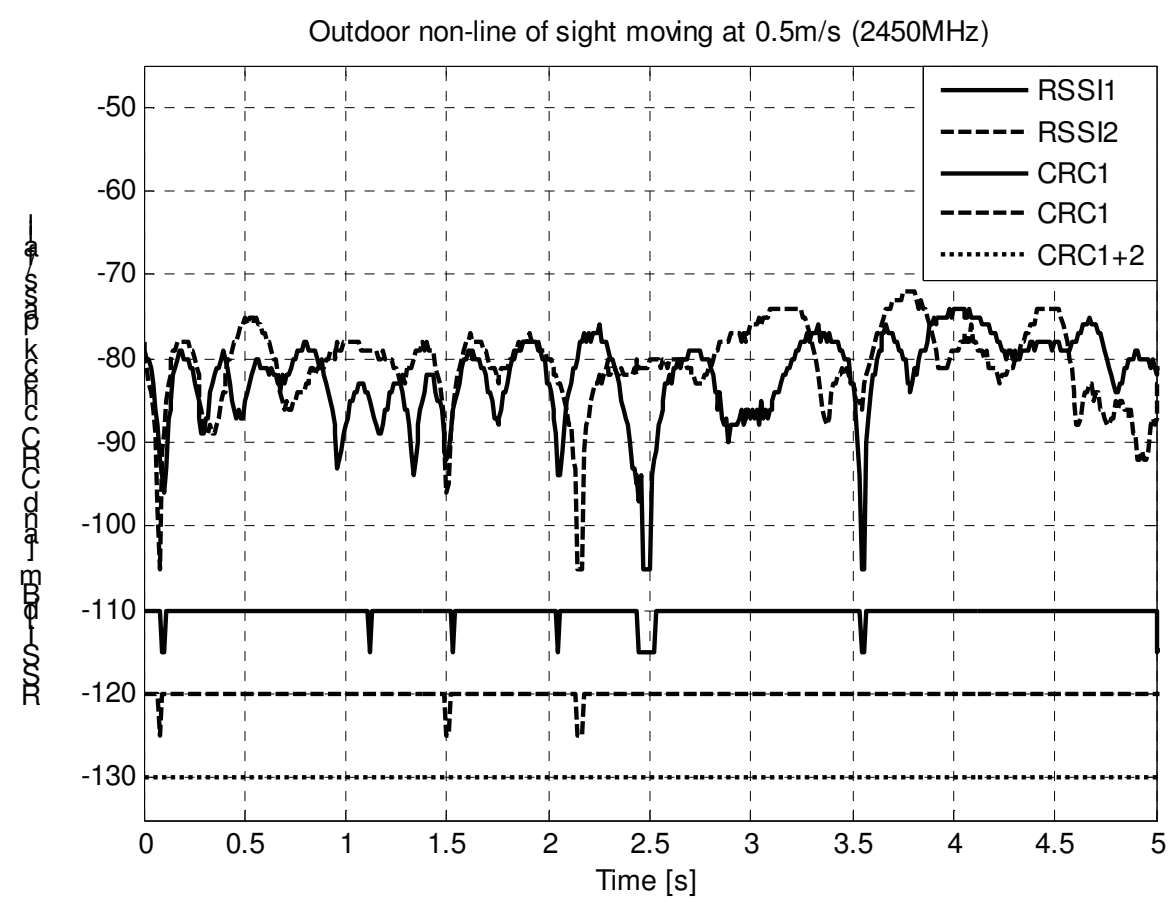

Figure 2 Indoor non-line of sight in a typical urban house using 1 transmitter and 2 receivers. The two receivers are spaced $15 \mathrm{~cm}$ apart and connected to the same microcontroller.

The spacing of the fades in time and space is related to the Doppler spread and is inversely proportional to the frequency of operation. In order for a dual receiver architecture to work, the two signals that are received need to be uncorrelated. This can be achieved by spacing the antenna apart, creating a 90 degree angle between the two antenna or a combination of both. For the dataset shown in Figure 2 the two RSSI profiles are not correlated and that for all the bursts (500) that were transmitted during the 5 second test period at least one of the receivers was not in a fade.

In the case of semi static channel or slow moving channel the fading is modeled as a slow fading system, where the entire burst of data can either be received or has been deleted by destructive interference.

It is important for semi static or slowly moving environments to include some kind of mechanism to avoid these deep fades in the time domain. The best approaches are described below.

- Time diversity: In its simplest form, time diversity is an acknowledge message with automatic retransmissions if acknowledge is not received. This works because the channel is constantly changing. Simply trying again later will in most cases results in a successful link.

- Frequency hopping: Works by constantly changing the frequency of operation and forcing the channel to change. This method works best in conjunction with the above "acknowledge and retry" scheme.

- Antenna diversity: As shown in Figure 2 antenna diversity is effective for semi static and slow moving channels, in many cases a delta between the two antenna can be in excess of $20 \mathrm{~dB}$. 
Again there is not a clear winner. Designers should consider the overall system requirement and make a choice on a case by case basis. However, in ultra low power sensor networks where retransmissions are "expensive" and the constant processing needs in the frequency hopping scheduler also might be too much, the clear winner becomes a receiver antenna diversity scheme. Typically the receiver is line powered, and it is acceptable to have dual receivers and have them on all the time.

\section{Fast fading channels}

If the speed of travel inside the channel increases to a point where a receiver can enter and exit a fade during the reception of a single burst of data, about it becomes a fast fading channel. The speed at which this happens is dependant on the burst length and the frequency operation. It is safe to assume that when the position of the transceiver is changed by less than $1 / 10$ of a wavelength during a single burst it is still of slow moving channel.

$$
\text { topspeed_2ms }=\frac{1 / 10 \lambda}{\text { burst_length }}=\frac{0.1 * 3 \cdot 10^{8}[\mathrm{~m} / \mathrm{s}]}{2.45 \cdot 10^{9}[\mathrm{~Hz}]^{*} 0.002 \mathrm{~s}}=6[\mathrm{~m} / \mathrm{s}]
$$

This gives an important consideration when making system tradeoffs between packet length and expected dynamic RF environments the system might operate in. Imagine the same calculation was done for a 64 byte packet at $1.2 \mathrm{~kb} / \mathrm{s}$. At the lower speed of $1.2 \mathrm{~kb} / \mathrm{s}$ it takes a full $426 \mathrm{~ms}$ to transmit just the data portion of the burst. When time is added for preamble and sync word, the total burst time is approximately $500 \mathrm{~ms}$.

$$
\text { topspeed_0.5s }=\frac{1 / 10 \lambda}{\text { burst_length }}=\frac{0.1 * 3 \cdot 10^{8}[\mathrm{~m} / \mathrm{s}]}{2.45 \cdot 10^{9}[\mathrm{~Hz}] * 0.5 \mathrm{~s}}=0.024[\mathrm{~m} / \mathrm{s}]
$$

This shows that lowering the bit rate to achieve the promised increased sensitivity has severe drawbacks when considering that the RF channel could change many times during a single burst.

To survive sub-frame bit deletions system like GSM/CDMA employ complicated forward error correction (FEC), data interleaving algorithm that require significant processing power to decode. Most low power wireless transceivers, like Zigbee, Bluetooth, ANT, do not employ FEC because of the processing power required and the fact that most low power networks are focused on static or semi-static environments.

\section{Coexistence}

The $2.45 \mathrm{GHz}$ ISM band is one of the most popular bands for unlicensed wireless systems to operate in; this leads to issue with coexistence. Coexistence is the techniques involved in making multiple autonomous RF communications systems operate in the same spectrum at the same time. For most low power wireless sensors nodes coexistence becomes a matter of surviving the environment which it is installed in. The reason for this is that most other systems transmit at significantly higher output. The three most prevalent systems are.

1. Bluetooth ${ }^{\mathrm{TM}}$

a. Frequency hopping spread spectrum, 1600 hops per second, radio does NOT perform a clear channel assess, it just transmits.

2. Wireless LAN (WIFI)

a. Advance digital modulation (CDMA and or OFDM). The channels can be up to $20 \mathrm{MHz}$ wide and does not move. Each radio performs a clear channel asses before each transmit. The modulation type of WIFI looks like noise to most low power radios and can cause the overall system to perform very poorly. 
3. Microwave Ovens (both commercial and residential)

a. Simple TWT (Traveling wave Tube) system for heating. The output power of the TWT is in excess of $1 \mathrm{KW}$ and overtime the seals on the ovens deteriorate leaving a residual signal of $1 \mathrm{~W}$ or more in the area of an operating oven. The RF signal follows the phase of the AC supply current and the carrier frequency is voltage dependant. This all results in narrowband high power noise moving randomly throughout the $2.4 \mathrm{GHz}$ band during operation.

Due to these reasons it is imperative that even the simplest low power systems incorporate some type of collision detection and avoidance.

\section{System Level Performance Techniques}

Beyond the inherent performance characteristics in the physical layer (the hardware) of the RF architecture, silicon designers imbed firmware in the MAC (medium access control) of the radio processor system to allow features in the HAL (hardware abstraction layer) to improve and optimize radio performance. The goal of these features is to get the message through on the first transmission. Packet error rate is a common benchmark for message centric wireless systems Any retry due to a failure to complete the link impacts the overall performance and efficiency of the communication network. The performance can be measured by the latency to complete the link (measured in time typically milliseconds to minutes), battery loss in mAh (milliamp hours) typically in weeks or months, and increased unwanted radio energy which adds to the noise floor. System architects employ CSMA (listen before talk), RSSI (receive signal strength indicator), Auto calibration storage values and MAC layer CSMA (listen before talk) features to allow multiple radio transmitters to operate in a single frequency channel by requiring the receiver to listen for other radio traffic on the channel and passing control or allowing the transmitter to send data stored in the FIFO buffer transmitter.

- CSMA (listen before talk) - The receiver is set up to sweep the channel and see if there is any other radio transmitting data. When there is a quiet time slot, then it will allow the radio to switch on, calibrate and send data. CSMA is an industry accepted best practice to prevent packet error loss.

- RSSI - The RSSI values deviation shown in figure 2 are dynamic values. When measured over time, the receiver can be set for specific minimum acceptable thresholds so that the transmitter does not have to increase power levels if lower power levels will suffice. A good analogy is accepted practice of whispering in a library to allow efficient radio traffic. As long as the signal level remains low, there is less distraction and minimal energy is expended. Conversely, the radio receiver sensitivity can be reduced reducing current consumption and improving battery life.

- Auto calibration- Radios typically must calibrate every time they transmit due to slight changes in crystal frequency, multipath between the transmitter and the receiver, and ambient temperature shifts during a 24 hour period. Over time, frequently used calibration settings can be stored in a register to eliminate an excessive amount of time and current consumption. The cumulative effect of eliminating the requirement to calibrate hundreds or thousands of transmissions per day with auto calibration is a huge savings in battery life. 


\section{Solar power use case of low power radio technology}

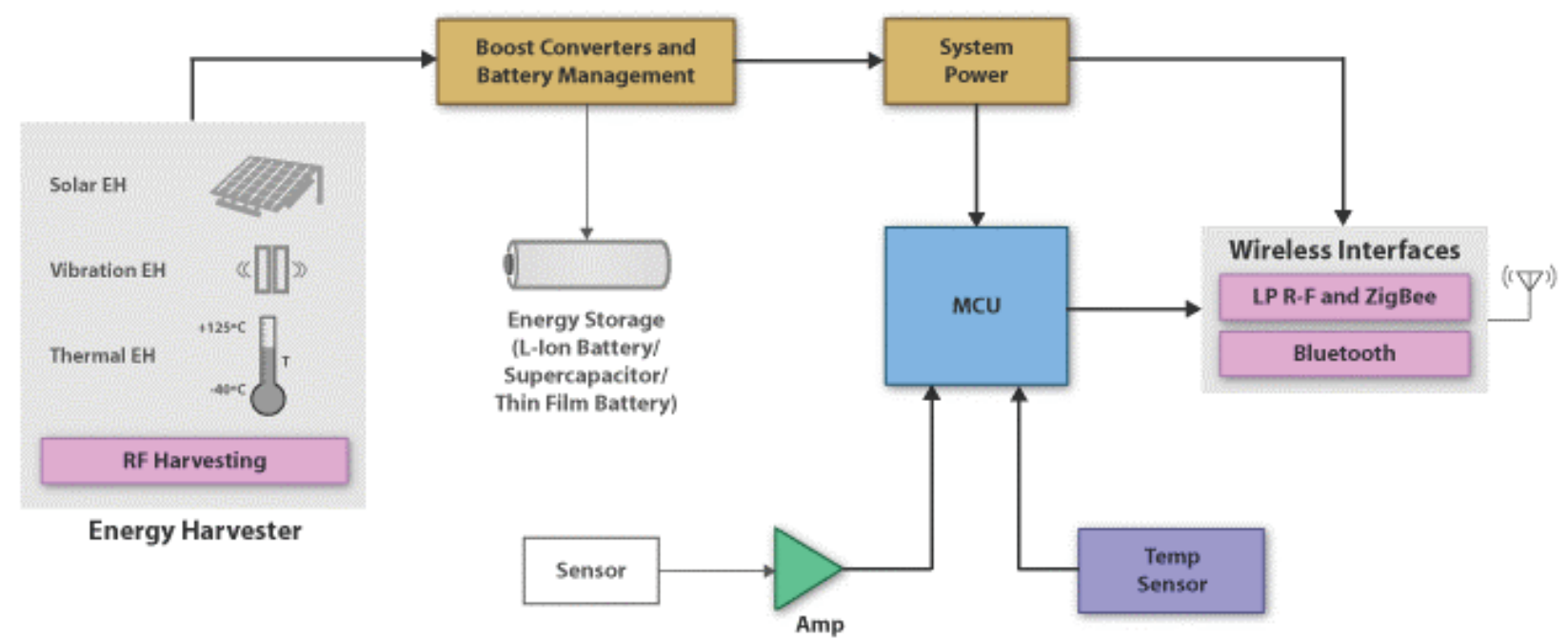

\begin{tabular}{|ll|}
\hline LEGEND & $\square$ Logic \\
$\square$ Processor & $\square$ Power \\
$\square$ Interface & $\square$ ADC/DAC \\
$\square$ RF/IF & $\square$ Clocks \\
$D$ Amplifier & $\square$ Other \\
\hline
\end{tabular}

Using the best practice features described in this paper allows the integration of sensors and wireless technology to be powered by energy harvesting or energy scavenging devices like solar cells. Designers should consider the following recommendations:

- Use harvesters sized for the correct power requirements of the application and communication method. Small postage stamp size solar cells in series can now power sensors in remote applications without any battery.

- Manage current consumption through the entire signal path. Efficient wireless systems are typically off $95-99 \%$ of the time. They employ efficient dc to dc power management hardware and bulk capacitors to store energy until required for a packet transmission.

- Manage RF/Microprocessor transition states to eliminate costly current consumption in calibration cycle.

In conclusion, we have discussed the key characteristics of effective and efficient low power RF communication systems. Existing barrier to robust RF communication were presented with suggestions for ways to avoid or coexist with some of these barriers. Best practice features in existing low power RF systems to implement these practices in energy harvesting systems were discussed. The opportunities to design wireless sensor communications into new and existing applications have recently reached a tipping point where they are economical and reliable to use. 